\title{
Sensibilidade ao contexto na identificação de estados afetivos aplicados à educação: um mapeamento sistemático
}

Sandro Oliveira Dorneles - Instituto Federal do Rio Grande do Sul - pfsandro@yahoo.com.br Debora Nice Ferrari Barbosa - Universidade Feevale - deboranice@ feevale.br

Jorge Luís Victória Barbosa - Universidade do Vale do Rio dos Sinos - jbarbosa@ unisinos.br

Resumo: Estudos apontam como tendência o uso de lentes emocionais e a relevância das emoções ou estados afetivos para as interações educacionais. Aliado a isso, e considerando as potencialidades da computação ubíqua e afetiva, cresce o entendimento da importância do uso de informações contextuais na identificação de sinais afetivos e sociais. Dessa forma, esse estudo apresenta uma revisão da literatura sobre o uso de informações de contextos na identificação de estados afetivos em ambientes educacionais. Os resultados indicam que os trabalhos fazem uso de canais visuais $(50 \%)$ e fisiológicos (50\%) associados principalmente a informações de contexto, relacionadas a atividades e identidade na detecção de estados afetivos. A revisão identificou ainda, o uso de 27 diferentes estados afetivos com predominância na valência das emoções positivas como felicidade e satisfação ou negativas como frustração e decepção.

Palavras-chave: computação afetiva, sensibilidade ao contexto, estados afetivos, ambientes de aprendizagem.

\section{Sensitivity to context in the identification of affective states applied to education: a systematic mapping}

Abstract: Studies point to the tendency to use emotional lenses and the relevance of emotions or affective states to educational interactions. Allied to this, and considering the potential of ubiquitous and affective computing, there is a growing understanding of the importance of using contextual information in the identification of affective and social signals. Thus, this study presents a review of the literature on the use of contextual information in the identification of affective states in educational environments. The results indicate that the works make use of visual (50\%) and physiological (50\%) channels mainly associated with contextual information, related to activities and identity in the detection of affective states. The review also identified the use of 27 different affective states with predominance in the valence of positive emotions like happiness and satisfaction or negative ones like frustration and disappointment.

Keywords: affective computing, context-aware, affective states, learning environments.

\section{Introdução}

Emoção e motivação são elementos fundamentais no comportamento humano e afetam as relações e interações nas atividades do dia a dia. Estudos recentes na área da neurociência reforçam o papel das emoções nas tomadas de decisões, sejam elas simples ou complexas, interferindo diretamente na forma como o ser humano se relaciona. Segundo Rowe e Fitness (2018), as emoções despertam comportamentos de adaptação que permitem aos indivíduos resolver dificuldades interpessoais e intrapessoais. A experiência da emoção provoca e coordena mudanças na cognição, fisiologia e comportamento.

Assim como os estados emocionais, existem outros aspectos comuns e igualmente importantes nas relações humanas tais como estado de ânimo, postura interpessoal, atitudes e personalidade. Esses fenômenos afetivos aliados à emoção, são definidos por Scherer et al. (2000), como sendo estados afetivos. A identificação e o entendimento de como esses estados afetam as relações têm sido alvo de estudos em áreas como a saúde 
(Prabhu et al., 2019), entretenimento (Diaz et al., 2018) e educação (Troussas e Virvou, 2020).

O estudo de estados afetivos em sistemas computacionais é chamado de computação afetiva sendo definida por Picard (1997), como a capacidade dos computadores de reconhecerem as emoções humanas, permitindo assim, ampliar as possibilidades de interação humano-computador e personalizar o uso em diferentes sistemas de recomendações (Mizgajski e Morzy, 2019). Dessa forma, essa área da computação, busca entender e interpretar padrões de informações que possam representar computacionalmente os estados afetivos.

O principal desafio na construção de sistemas capazes de reconhecer os estados afetivos é identificar as sutilezas e alterações do comportamento humano. Segundo Jaques e Nunes (2013), existem diferentes reações ou canais de reconhecimento das emoções, tais como voz, comportamento observável, expressões faciais e sinais fisiológicos. No entanto, autores como Samy et al. (2018) e Lin et al. (2016) salientam a importância de um reconhecimento multimodal capaz de analisar diferentes parâmetros para a interpretação dos estados afetivos.

Nesse sentido, estudos apontam a importância do uso de informações de contexto na análise de afeto, a fim de interpretar os estados emocionais de forma mais precisa (Vlachostergiou et al., 2014; Moore, 2017). Sistemas sensíveis ao contexto, são sistemas inteligentes capazes de se adaptar às necessidades de usuários e ou ambientes para oferecer recursos personalizados. O contexto nesses sistemas é descrito por Dey (2001), como sendo qualquer informação que possa ser usada para caracterizar a situação de uma entidade, que pode ser uma pessoa, local ou objeto que é considerado relevante para a interação entre um usuário e um aplicativo, incluindo o usuário e os próprios aplicativos.

O armazenamento de informações de contexto para uso posterior, é reconhecido como históricos de contextos (Barbosa et al., 2018) e pode auxiliar na extração de padrões (Dupont et al., 2019), apoiando a previsão de contexto futuro (Da Rosa et al., 2016). A aplicação de informações de contexto, tem sido utilizada na avaliação de indicadores no reconhecimento de depressão, estresse pós-traumático (Stratou e Morency, 2017) e estresse mental (Bavaresco et al., 2020).

Yang e Damásio (2007) destacam ainda, a importância do afeto na educação, principalmente na atenção, memória e motivação do aluno. Assim, entender os diferentes estados afetivos e seus efeitos positivos ou negativos durante os processos de aprendizagem, é de fundamental importância para melhorar ações diagnósticas e de adaptação para o aluno. Nesse sentido, trabalhos como Cho e Casteñeda (2019) e Khan et al. (2019) apresentam pesquisas que buscam detectar emoções e adaptar estratégias pedagógicas em ambientes educacionais.

Revisões de estudos na área de computação afetiva aplicada à educação têm sido realizadas nos últimos anos. Reis et al. (2018) apresentam um mapeamento sistemático, em que buscam identificar o uso de estados afetivos e fatores sócio emocionais no desenvolvimento de ambientes de aprendizagem colaborativa, suportada por computador. Malekzadeah et al. (2014) apresentaram uma revisão de trabalhos que implementaram diferentes estratégias de gerenciamento de emoções e regulação emocional em sistemas de tutoria inteligente. Yadegaridehkordi et al. (2019) buscaram identificar domínios de aprendizagem, canais e métodos de reconhecimento de estados afetivos, além de teorias, modelos e estados emocionais utilizados. 
Por sua vez, Vlachostergiou et al. (2014) propõem uma reflexão sobre o uso de informações contextuais na computação afetiva, identificando quais informações são relevantes e como a integração, melhoraria o desempenho das estruturas multimodais no reconhecimento de estados afetivos. Dessa forma, esse trabalho tem como objetivo investigar o uso de informações de contexto na análise do comportamento afetivo humano e as conexões entre sensibilidade ao contexto e estados afetivos na adaptação dos processos de aprendizagem. Os resultados são apresentados através de um mapeamento sistemático da literatura, visando compreender como as informações de contexto têm sido utilizadas na inferência de estados afetivos e seu impacto nos processos de aprendizagem.

O estudo foi realizado a partir de 5 bases de dados e está estruturado em 4 seções. A seção 2 define a metodologia de pesquisa. A seção 3 comenta os resultados para cada questão de pesquisa. A seção 4 apresenta considerações e trabalhos futuros.

\section{Metodologia}

O método utilizado foi baseado nos procedimentos propostos (Petersen et al., 2008) e atualizados por Petersen et al. (2015) para promover um mapeamento de estudos primários, cuja execução foi composta das seguintes etapas: estabelecer as questões de pesquisa; definir o processo de busca; estabelecer os critérios para filtro dos resultados e executar as análises e classificação dos resultados.

\subsection{Questões de pesquisas}

O objetivo deste mapeamento foi verificar estudos relacionados à identificação de estados afetivos associados ao uso de informações de contexto na educação, como forma de adaptar e ou personalizar ações nos processos de aprendizagem. Desse modo, a Tabela 1 mostra: Questões de pesquisa Gerais (QG) e Questões de pesquisa Focais (QF).

\begin{tabular}{c|l}
\multicolumn{1}{c}{ Tabela 1: Questões de Pesquisa } \\
\hline ID & \multicolumn{1}{c}{ Questões de Pesquisa } \\
\hline QG1 $\begin{array}{l}\text { Quais informações de contexto e canais emocionais são usados como parâmetros para o reconhecimento dos } \\
\text { estados afetivos? }\end{array}$ \\
QG2 $\begin{array}{l}\text { Do ponto de vista da aprendizagem há indicação das ações adaptadas ao estudante a partir do uso de contextos } \\
\text { e estados afetivos detectados? }\end{array}$ \\
QF1 Quais recursos computacionais são utilizados para a obtenção dos estados afetivos? \\
QF2 Quais estados afetivos são considerados nos estudos?
\end{tabular}

\subsection{Definição de strings de busca}

No sentido de identificar palavras-chave para construção da string de busca foram realizadas pesquisas prévias em artigos das áreas de computação afetiva, sensibilidade ao contexto e aprendizagem. Dessa forma, foram definidas como palavras-chave: context awareness, affective computing, emotion, affective state, learning e education. As bases utilizadas na busca foram escolhidas pela reconhecida relevância na área da computação, são elas: ACM Digital Library, IEEE Xplore Digital Library, Science Direct, Springer e Scopus.

A construção da string levou em conta sinônimos e termos relacionados com as palavras-chaves, e uso de expressões booleanas. Assim, foi definida a string de consulta como: ("context-aware" or "context awareness" or "ubiquitous computing" or "pervasive computing") AND ("Emotion" or "Affective" or "Affective State") AND ("learning" or "education"). No entanto, para cada base foi necessário adaptar a string, para buscas no título, resumo e palavras-chave.

V. $18 \mathrm{~N}^{\mathrm{o}} 1$, julho, 2020 RENOTE DOI: 


\subsection{Critérios de exclusão e inclusão}

Os critérios de seleção para inclusão das publicações foram: i) CI1. Publicações a partir de 2009 inclusive; ii) CI2. Estudos escritos em inglês; iii) CI3. Trabalhos que apresentassem evidências relacionadas ao tema da pesquisa; iv) CI4. As publicações deveriam apresentar resultados de experimentos. Os critérios de exclusão utilizados foram: i) CE1. Artigos duplicados em diferentes bases de pesquisa; ii) CE2. Teses, dissertações, livros e revisões.

Os critérios CI1 e CI2 foram aplicados diretamente nos mecanismos de busca. Em seguida, foi aplicado o critério CE1 à procura de artigos duplicados. Na sequência realizou-se a leitura de títulos e resumos dos estudos selecionados. Os resultados são apresentados na Figura 1.

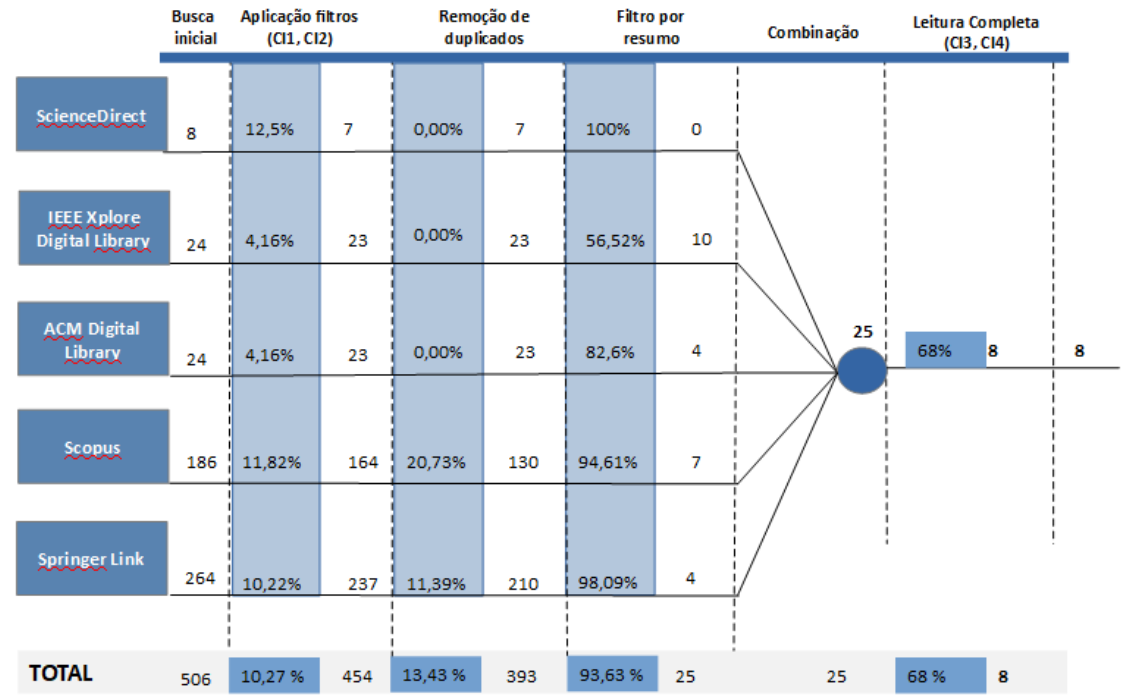

Figura 1: Etapas dos processos de pesquisa.

Após a leitura completa dos trabalhos selecionados foram encontradas nas referências dois novos artigos que atendiam aos critérios de inclusão estabelecidos no protocolo de busca. Os trabalhos foram localizados utilizando buscas manuais no Scholar Google. A Tabela 2 apresenta a relação dos artigos mapeados organizados por id, referência, base de dados, canais emocionais e parâmetros utilizados.

Tabela 2: Relação dos artigos mapeados.

\begin{tabular}{|c|c|c|c|c|}
\hline ID & Referência & Base & Canal & Parâmetro \\
\hline R01 & Shen et al. (2009) & Manual & Fisiológico & $\begin{array}{l}\text { EEG do cérebro, GSR e pressão } \\
\text { sanguínea. }\end{array}$ \\
\hline $\mathrm{R} 02$ & Castellano et al. (2010) & $\mathrm{ACM}$ & Visual & Face e postura \\
\hline R03 & Rhee et al. (2010) & IEEE & Fisiológico & Dilatação da pupila \\
\hline R04 & Kuo e Tseng (2011) & IEEE & Fisiológico & GSR \\
\hline R05 & Alepis e Virvou (2012) & Springer & $\begin{array}{l}\text { Comportamento observável } \\
\text { e vocal }\end{array}$ & Face, postura e fala \\
\hline R06 & Saneiro et al.(2014) & Manual & $\begin{array}{l}\text { Visual e comportamento } \\
\text { observável }\end{array}$ & Face e postura \\
\hline R07 & Shminan e Othman (2015) & IEEE & Visual & Face e postura \\
\hline R08 & Santos et al. (2016) & Scopus & Fisiológico & $\begin{array}{l}\text { Frequência } \\
\text { respiratória e GSR. }\end{array}$ \\
\hline R09 & Zheng et al. (2018) & IEEE & Vocal e visual & Face, gestos e fala \\
\hline $\mathrm{R} 10$ & Uria-Rivas et al.(2019) & Scopus & Fisiológico & $\begin{array}{l}\text { Frequência } \\
\text { respiratória e GSR. }\end{array}$ \\
\hline
\end{tabular}




\section{Resultados}

Nesta seção é apresentado o detalhamento dos artigos selecionados, nas subseções a seguir, são exibidos os resultados para cada questão de pesquisa.

\subsection{QG1 Quais informações de contexto e canais emocionais são usados como parâmetros para o reconhecimento dos estados afetivos?}

O uso de sistemas sensíveis ao contexto na identificação de estados afetivos exige uma abordagem multimodal, na identificação das reações emocionais e nas interações que podem ser utilizadas como informações de contexto.

Considerando a identificação dos estados afetivos sob a perspectiva da computação afetiva, pode-se categorizar como ambientes computacionais, que possuem capacidade de reconhecer, expressar, ou ainda reconhecer e expressar emoções. Esses sistemas, utilizam reações sensoriais ou fisiológicas dos usuários no reconhecimento dos estados afetivos. As reações identificadas nesse mapeamento para reconhecimento dos estados afetivos foram classificadas como vocal, comportamento observável, visual e fisiológicas conforme Tabela 2.

Um número considerável de trabalhos (50\%) indica o uso de canais fisiológicos para identificação das reações afetivas, tendo como parâmetros frequência cardíaca, respiratória, pressão sanguínea e resposta galvânica da pele ou Galvanic Skin Response (GSR) que reflete alterações na condutância e temperatura da pele. Os trabalhos de Shiminan e Othman (2015) e Castellano et al. (2010) utilizam canais visuais usando como parâmetros a identificação de expressões da face e ou postura e gestos corporais.

Nenhum dos trabalhos apresenta uso exclusivo de sinal vocal ou comportamento observável no entanto, essas reações são usadas em conjunto de forma multimodal no estudo de Alepis e Virvou (2012) que utiliza como parâmetro o reconhecimento da face, gestos e voz. O trabalho de Zheng et al. (2018) apresenta o uso multimodal no reconhecimento vocal e visual tendo como indicadores face, postura e fala. Saneiro et al. (2014) fazem uso dos canais visual e comportamento observável utilizando como critério a identificação da face e postura do usuário.

Para a análise da sensibilidade ao contexto, foram consideradas nesse mapeamento informações de contexto definidas por Dey (2001) como identidade, localização, atividades e tempo. Foram identificados o uso dos quatro tipos de informações de contexto. Setenta por cento dos artigos utilizaram o contexto atividades de entidades (R09, R10, R02, R03, R08, R05, R06) em que foram usadas informações como ações de usuários, câmeras e microfones (R09), rastreamento de sensores (R10), logs de usuários (R02, R08, R05, R06) e iluminação de ambiente (R03). A característica identidade, foi utilizada em 50\% dos trabalhos (R07, R04, R05, R01, R06), onde foram consideradas informações sobre o perfil do aluno como dados demográficos, idade, sexo, estilo de aprendizagem e de interação, ocupação e traços de personalidade.

Já o contexto localização foi identificado em 30\% dos trabalhos (R09, R03, R08), nos quais foram utilizados sensores de GPS. O tempo foi usado como contexto em $30 \%$ dos trabalhos (R02, R08, R06), nos quais foram coletados dados da data e hora de eventos das entidades. As relações entre os canais de reconhecimento afetivo e os contextos podem ser vistos na Figura 2. Nesse sentido, determinados trabalhos utilizam mais de uma característica de contexto, combinando-as na identificação dos estados afetivos.

Existe uma relação entre a sensibilidade ao contexto e estados afetivos (Moore, 2017), na medida em que o comportamento emocional forma um elemento importante no V. $18 \mathrm{~N}^{\mathrm{o}} 1$, julho, 2020 RENOTE DOI: 
contexto de um indivíduo, o contexto também afeta seu estado emocional. Diante disso, a Figura 2 mostra que as informações de contexto mais utilizadas nos trabalhos são a identidade e atividades e estão relacionadas com canais fisiológicos e multimodais. Alguns trabalhos apontam ainda, o arranjo de diferentes informações de contexto, aumentado assim, a complexidade para medi-las, armazena-las e processa-las, refletindo assim, a natureza complexa e dinâmica do uso de contextos na computação afetiva (Moore 2017).

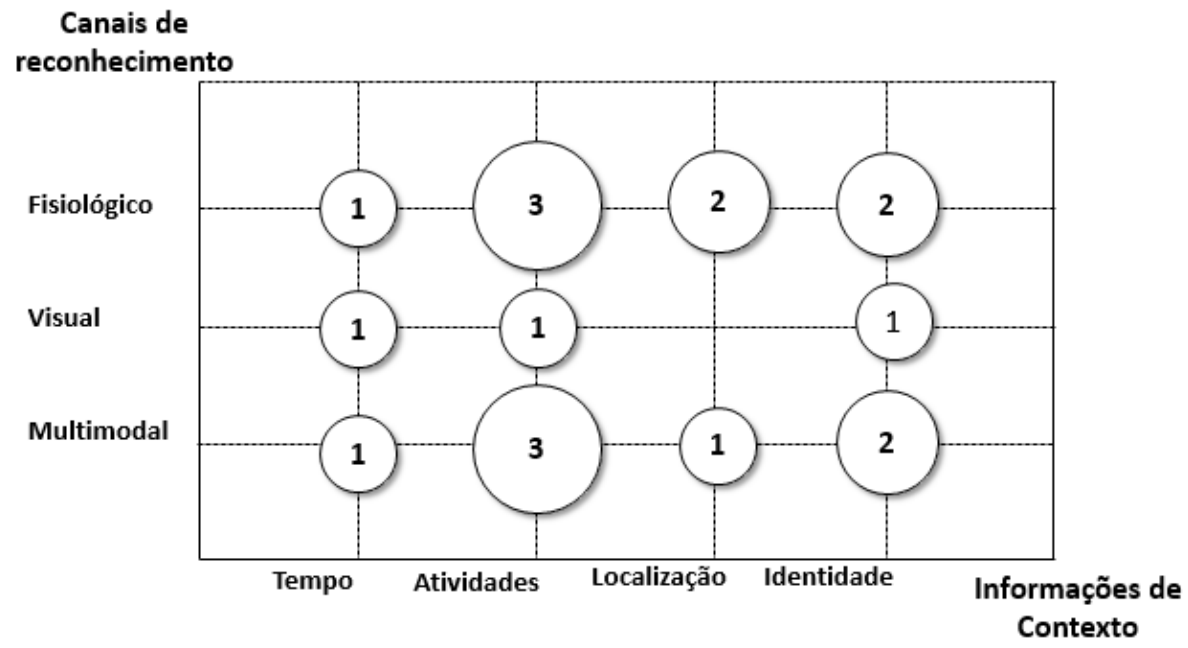

Figura 2: Informações de contexto utilizadas.

3.2 QG2 - Do ponto de vista da aprendizagem há indicação de ações adaptadas ao estudante a partir do uso de contextos e estados afetivos detectados?

A maioria dos artigos (60\%) apresentam ações de regulação dos estados afetivos no ambiente educacional. Os trabalhos apresentaram intervenções como ajuste de conteúdo e nível de dificuldade (Zheng et al., 2018; Kuo e Tseng, 2011; Shen et al., 2009), recomendação de materiais relevantes (Shminan e Othman, 2015), regulação emocional (Uria-Rivas et al., 2019) e apoio afetivo (Santos et al., 2016). O trabalho de Castellano et al. (2010) utiliza o estado afetivo para adaptar a interface de comunicação com o aluno. Por sua vez, Rhee et al. (2010) faz apenas regulação do ambiente para melhorar a aferição das emoções.

Apesar do aumento nos últimos anos de trabalhos relacionados à computação afetiva e uso de informações de contexto no reconhecimento de estados emocionais, devese reconhecer que ainda existem desafios em termos de modelagem eficaz do cérebro humano e na mensuração de estados afetivos (Moore et al., 2017). Os resultados corroboram a percepção de Moore (2017), onde é possível perceber, que um número significativo de trabalhos (40\%) concentrou esforços em sistemas e instrumentos de reconhecimento dos estados afetivos e não no uso dessas informações na regulação ou adaptação em ambiente de aprendizagem.

\subsection{QF1 - Quais recursos computacionais são utilizados para a obtenção dos estados afetivos?}

Um dos principais desafios na identificação da emoção é o uso de recursos não intrusivos que possam ser utilizados no mundo real nesse caso específico, em um ambiente educacional. Os recursos utilizados nos trabalhos analisados estão diretamente ligados aos canais e parâmetros coletados. Os trabalhos de Zheng, et al. (2018) e Alepis e Virvou (2012) utilizaram uma combinação de câmeras e microfone para captura da face 
e fala do usuário. Os artigos de Castellano et al. (2010) e Rhee et al. (2010) utilizam somente câmeras para captura da face e postura corporal.

Shminan e Othman (2015) e Saneiro et al. (2014) utilizaram o módulo Kinect da Microsoft, composto por câmera 3D, sensor de profundidade e microfone, capaz de identificar expressões faciais e gestos. Uria-Rivas et al. (2019), Kuo e Tseng (2011) e Santos et al. (2016) usaram sensores fisiológicos como eletrocardiograma ECG e axiômetro de pulso para identificação da frequência cardíaca, sensor de frequência respiratória, GSR para resposta galvânica da pele e sensores para temperatura corporal. Shen et al. (2009) utilizaram sensores de condutância da pele, um fotopletismógrafo para medir a pressão do volume sanguíneo e um sensor eletroencefalográfico que mede a atividade de EEG do cérebro.

Os resultados indicam que os principais recursos utilizados são sensores para medir sinais fisiológicos do indivíduo (50\%) e diferentes modelos de câmeras e microfones para captura de imagens e áudios $(50 \%)$.

\subsection{QF2 - Quais estados afetivos são considerados no estudo?}

Os estados emocionais identificados nos trabalhos foram categorizados como estados afetivos conforme proposto por Scherer et al. (2000). A Figura $3 \mathrm{em}$ forma de nuvem de palavras, representa todos os fenômenos afetivos identificados nos artigos. $\mathrm{O}$ tamanho da fonte de cada estado afetivo está diretamente relacionado ao número de publicações em que foi considerado. Os termos mais relevantes representados na Figura 3 fazem referência a estados positivos, negativos, frustração e felicidade.

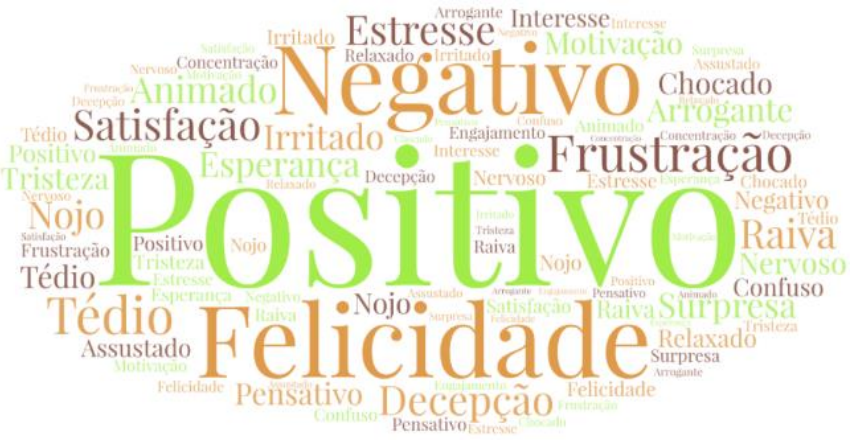

Figura 3: Estados afetivos.

O trabalho de Zheng et al. (2018) utilizou como base, estados emocionais cognitivos como tédio, confusão, felicidade, frustração, concentração e surpresa para identificar o nível de atenção. O artigo de Shminan e Othman (2015) buscou aferir o nível de motivação durante as aulas, a fim de aumentar o grau de engajamento. O estresse é considerado por Uria-Rivas et al. (2019), que investigou através de sensores mudanças no estado emocional do usuário identificando situações estressantes que podem prejudicar o desempenho do aluno.

O estudo de Alepis e Virvou (2012) analisou estados como felicidade, raiva, nojo, tristeza, surpresa e neutro. Shen et al. (2009) consideraram estados como engajamento, confusão, frustração, tédio, esperança, satisfação e decepção.

Grande parte dos trabalhos analisados (50\%) utilizaram uma abordagem dimensional, formada pela valência (positiva ou negativa) e excitação (calma, excitado) dos fenômenos afetivos (Scherer et al., 2000). Os trabalhos de Castellano et al. (2010), Kuo e Tseng (2011) e Santos et al. (2016) buscaram identificar a valência dos estados afetivos relacionados a situações agradáveis como felicidade, satisfação, alegria e 
esperança, ou estados negativos que fazem referência a tristeza, irritação, frustração e decepção. Os trabalhos de Rhee et al. (2010) e Saneiro et al. (2014) consideraram somente o grau de estado afetivo (positivo ou negativo) e de excitação.

O resultado do mapeamento identificou 27 estados afetivos diferentes. Desses, o principal foco de identificação nos trabalhos foram felicidade (30\%), frustração (30\%) e valências negativas ou positivas $(50 \%)$.

\section{Considerações}

Este trabalho se propôs a identificar em bases de dados científicas, artigos que pudessem permitir a análise de pesquisas relacionadas ao uso de informações de contextos associados a identificação de estados afetivos na conexão e adaptação de processos de aprendizagem.

Os resultados apontam que o foco principal dos trabalhos é o aperfeiçoamento de modelos de reconhecimento das emoções. Desses trabalhos, $40 \%$ não fazem uso das informações de estados afetivos para adaptação ou personalização da aprendizagem. Em relação as reações emocionais, os canais visuais (50\%) utilizados sozinhos ou de forma multimodal e canais fisiológicos $(50 \%)$ têm sido os principais recursos na identificação dos estados afetivos, estão associados principalmente a informações de contexto como atividades $(70 \%)$ e identidade de entidades (50\%). Quanto aos estados afetivos identificados parte relevante dos trabalhos (50\%) considerou valências positivas e negativas das emoções.

A integração da computação afetiva e informações de contextos oferece o potencial de melhorar modelos de reconhecimento afetivo permitindo assim, o uso de sistemas de recomendação ou regulação em ambientes formais e informais de educação. Outros temas como educação personalizada com base no contexto e estados afetivos, sistemas de regulação afetiva, uso de wereable e históricos de contextos na identificação de estados afetivos, são tópicos que podem ser explorados em trabalhos futuros.

\section{Referências Bibliográficas}

ALEPIS, Efthymios; VIRVOU, Maria. Multimodal object oriented user interfaces in mobile affective interaction. Multimedia Tools and Applications, v. 59, n. 1, p. 41-63, 2012.

BARBOSA, Jorge et al. TrailCare: An indoor and outdoor Context-aware system to assist wheelchair users. International Journal of Human-Computer Studies, v. 116, p. 1-14, 2018.

BAVARESCO, Rodrigo et al. Design and evaluation of a context-aware model based on psychophysiology. Computer Methods and Programs in Biomedicine, v. 189, 105299, 2020.

CASTELLANO, Ginevra et al. Inter-ACT: An affective and contextually rich multimodal video corpus for studying interaction with robots. In: Proceedings of the 18th ACM international conference on Multimedia. ACM, 2010. p. 1031-1034.

CHO, Moon-Heum; CASTAÑEDA, Daniel A. Motivational and affective engagement in learning Spanish with a mobile application. System, v. 81, p. 90-99, 2019.

DA ROSA, João H. ; BARBOSA, Jorge L.V. ; RIBEIRO, Giovane D. . ORACON: An adaptive model for context prediction. Expert Systems with Applications, v. 45, p. 5670, 2016. 
DEY, Anind K. Understanding and using context. Personal and ubiquitous computing, v. 5, n. 1, p. 4-7, 2001.

DIAZ, Yancarlos et al. Towards an affective video recommendation system. In: 2018 IEEE International Conference on Pervasive Computing and Communications Workshops (PerCom Workshops). IEEE, 2018. p. 137-142.

DUPONT, Daniel A. ; MOTA, Bruno ; BARBOSA, Jorge L. V. . CHSPAM: a multidomain model for sequential pattern discovery and monitoring in contexts histories. Pattern Analysis and Applications, v. 1, p. 1-10, 2019.

IMMORDINO-YANG, Mary Helen; DAMASIO, Antonio. We feel, therefore we learn: The relevance of affective and social neuroscience to education. Mind, brain, and education, v. 1, n. 1, p. 3-10, 2007.

JAQUES, Patrícia A.; NUNES, Maria Augusta SN. Ambientes Inteligentes de Aprendizagem que inferem, expressam e possuem emoções e personalidade. Jornada de Atualização em Informática na Educação, v. 1, n. 1, p. 30-81, 2013.

KHAN, Farman Ali et al. Automatic Student Modelling for Detection of Learning Styles and Affective States in Web Based Learning Management Systems. In: IEEE Access, v. 7, p. 128242-128262, 2019.

KUO, Yu-Chen; TSENG, Chih-Chi. Recognizing the emotion of learners by physiological sensors to improve english learning performance. In: 2011 4th International Conference on Biomedical Engineering and Informatics (BMEI). IEEE, 2011. p. 2152-2156.

LIN, Hao-Chiang Koong et al. Construction of multi-mode affective learning system: taking affective design as an example. Journal of Educational Technology \& Society, v. 19, n. 2, p. 132-147, 2016.

MALEKZADEH, Mehdi; SALIM, Siti Salwah; MUSTAFA, Mumtaz Begum. Towards Integrating Emotion Management Strategies in Intelligent Tutoring System Used by Children. In: International Symposium on Pervasive Computing Paradigms for Mental Health. Springer, Cham, 2014. p. 41-50.

MIZGAJSKI, Jan; MORZY, Mikołaj. Affective recommender systems in online news industry: how emotions influence reading choices. User Modeling and User-Adapted Interaction, v. 29, n. 2, p. 345-379, 2019.

MOORE, Philip. Do We Understand the Relationship between Affective Computing, Emotion and Context-Awareness? Machines, v. 5, n. 3, p. 16, 2017.

PETERSEN K., VAKKALANKA, S., KUZNIARZ, L., Guidelines for Conducting Systematic Mapping Studies in Software Engineering: An Update. Information and Software Technology, vol. 64, 2015, pp. 1-18

PETERSEN, Kay et al. Systematic mapping studies in software engineering. In: 12th International Conference on Evaluation and Assessment in Software Engineering, vol. 17, p. 1-10, 2008.

PICARD, R. W. Affective Computing. Cambridge: MIT Press. 1997

PRABHU, Vishnunarayan G. et al. An Affective Computing in Virtual Reality Environments for Managing Surgical Pain and Anxiety. In: 2019 IEEE International Conference on Artificial Intelligence and Virtual Reality (AIVR). IEEE, 2019. p. 2352351. 
REIS, Rachel Carlos Duque et al. Affective states in computer-supported collaborative learning: Studying the past to drive the future. Computers \& Education, v. 120, p. 29$50,2018$.

RHEE, Phill Kyu; NAM, Mi Young; WANG, Liang. Pupil location and movement measurement for efficient emotional sensibility analysis. In: The 10th IEEE International Symposium on Signal Processing and Information Technology. IEEE, 2010. p. 1-6.

ROWE, Anna D.; FITNESS, Julie. Understanding the role of negative emotions in adult learning and achievement: A social functional perspective. Behavioral Sciences, v. 8, n. 2, p. 27, 2018.

SAMY, Ahmed E.; EL-BELTAGY, Samhaa R.; HASSANIEN, Ehab. A Context Integrated Model for Multi-label Emotion Detection. Procedia computer science, v. 142, p. 61-71, 2018.

SANEIRO, Mar et al. Towards emotion detection in educational scenarios from facial expressions and body movements through multimodal approaches. The Scientific World Journal, v. 2014, 2014.

SANTOS, Olga C. et al. Toward interactive context-aware affective educational recommendations in computer-assisted language learning. New Review of Hypermedia and Multimedia, v. 22, n. 1-2, p. 27-57, 2016.

SCHERER, Klaus R. et al. Psychological models of emotion. The neuropsychology of emotion, v. 137, n. 3, p. 137-162, 2000.

SHEN, Liping; WANG, Minjuan; SHEN, Ruimin. Affective e-learning: Using "emotional" data to improve learning in pervasive learning environment. Journal of Educational Technology \& Society, v. 12, n. 2, p. 176-189, 2009.

SHMINAN, Ahmad Sofian; OTHMAN, Mohd Kamal. Dynamic student assessment to advocate personalized learning plan. In: 2015 International Conference on Information Technology Systems and Innovation (ICITSI). IEEE, 2015. p. 1-6.

STRATOU, Giota; MORENCY, Louis-Philippe. MultiSense-Context-aware nonverbal behavior analysis framework: A psychological distress use case. IEEE Transactions on Affective Computing, v. 8, n. 2, p. 190-203, 2017.

TROUSSAS, Christos; VIRVOU, Maria. Affective Computing and Motivation in Educational Contexts: Data Pre-processing and Ensemble Learning. In: Advances in Social Networking-based Learning. Springer, Cham, 2020. p. 69-98.

URIA-RIVAS, R. et al. Impact of Physiological Signals Acquisition in the Emotional Support Provided in Learning Scenarios. Sensors, v. 19, n. 20, 4520, 2019.

VLACHOSTERGIOU, Aggeliki; CARIDAKIS, George; KOLLIAS, Stefanos. Investigating context awareness of affective computing systems: a critical approach. Procedia Computer Science, v. 39, p. 91-98, 2014.

YADEGARIDEHKORDI, Elaheh et al. Affective computing in education: A systematic review and future research. Computers \& Education, v. 142, 103649, 2019.

ZHENG, Jianbo et al. Cognition-Based Context-Aware Cloud Computing for Intelligent Robotic Systems in Mobile Education. In: IEEE Access, v. 6, p. 49103-49111, 2018. 